\title{
TOTAL NORMALITY AND THE HEREDITARY PROPERTY
}

\author{
RICHARD E. HODEL ${ }^{1}$
}

1. Introduction. In 1953 Dowker [4] introduced a new normality condition which he called total normality. He proved that every perfectly normal space is totally normal, and every totally normal space is completely normal. In this paper we prove further results about totally normal spaces. In particular, we show that if $X$ is a totally normal space having a topological property $P$ which satisfies certain axioms, then every subset of $X$ has property $P$. Two main results are then as follows: (1) every totally normal paracompact space is hereditarily paracompact; (2) every totally normal collectionwise normal space is hereditarily collectionwise normal.

The reader is referred to E. Michael's papers [5], [6] for definitions concerning open covers, refinements, closure preserving collections, locally finite collections, and paracompactness, and to R. H. Bing's paper [1] for definitions concerning discrete collections and collectionwise normality. A topological space $X$ is completely normal if every subset of $X$ is normal. A topological space $X$ is hereditarily paracompact (hereditarily collectionwise normal) if every subset of $X$ is paracompact (collectionwise normal).

Let $X$ be a normal space. $X$ is said to be perfectly normal if every open subset of $X$ is an $F_{\sigma}$ in $X$, and $X$ is said to be totally normal if every open subset $U$ of $X$ can be written as a locally finite (in $U$ ) collection of open $F_{\sigma}$ subsets of $X$. All spaces in this paper are assumed to be Hausdorff.

2. The axioms for $P$. Let $P$ be a topological property satisfying these axioms.

Axiom 1. If $X$ is any topological space such that every open subset of $X$ has property $P$, then every subset of $X$ has property $P$.

Axiom 2. If $X$ is any topological space having property $P$, and $U$ is an $F_{\sigma}$ subset of $X$, then $U$ has property $P$.

Axiom 3. If $X$ is any topological space, and $\left\{V_{\alpha}: \alpha\right.$ in $\left.A\right\}$ is a locally finite open cover of $X$ such that for all $\alpha$ in $A, \bar{V}_{\alpha}$ has property $P$, then $X$ has property $P$.

We then have the following theorem.

Presented to the Society, September 3, 1965; received by the editors June 2, 1965.

1 Supported by NSF Grant GF2065. 
Theorem 1. If $X$ is a totally normal space having property $P$, then every subset of $X$ has property $P$.

Proof. Let $U$ be a subset of $X$; we want to show that $U$ has property $P$. By Axiom 1, we may assume that $U$ is open. Since $X$ is totally normal, $U=U\left\{U_{\alpha}: \alpha\right.$ in $\left.A\right\}$, where each $U_{\alpha}$ is an open $F_{\sigma}$ in $X$, and the collection $\left\{U_{\alpha}: \alpha\right.$ in $\left.A\right\}$ is locally finite in $U$. Since every totally normal space is completely normal $[4$, p. 276], the space $U$ is normal. Hence the locally finite open cover $\left\{U_{\alpha}: \alpha\right.$ in $\left.A\right\}$ of $U$ has, by $\left[2\right.$, p. 71], an open refinement $\left\{V_{\alpha}: \alpha\right.$ in $\left.A\right\}$ such that for all $\alpha$, $F_{\alpha}\left(F_{\alpha}=\right.$ closure of $V_{\alpha}$ in $\left.U\right)$ is contained in $U_{\alpha}$. Now for all $\alpha$ in $A$, $F_{\alpha}$ is an $F_{\sigma}$ in $X$, and so by Axiom $2, F_{\alpha}$ has property $P$. It then follows by Axiom 3 that $U$ has property $P$.

3. Paracompactness. Paracompactness is a topological property satisfying Axioms 1-3. Axiom 1 is due to Dieudonné [2, p. 68]; Axiom 2 is due to Michael [5, p. 835]; Axiom 3 is due to Michael [5, p. 831]. This proves Theorem 2. Note that Theorem 2 is a generalization of Dowker's result [3, p. 643] that every perfectly normal paracompact space is hereditarily paracompact.

Theorem 2. Every totally normal paracompact space is hereditarily paracompact.

Dowker proved [4, p. 273] that every hereditarily paracompact space is totally normal. Combining this result with Theorem 2 gives the following theorem.

Theorem 3. Let $X$ be a paracompact space. Then $X$ is hereditarily paracompact if and only if $X$ is totally normal.

4. Collectionwise normality. Collectionwise normality is a topological property satisfying Axioms 1-3. Axiom 1 follows from Lemma 1 below; Axiom 2 is due to Sedivá [8, p. 53]; Axiom 3 follows from Lemma 2 below. This proves Theorem 4 .

Theorem 4. Every totally normal collectionwise normal space is hereditarily collectionwise normal.

Leмma 1. If every open subset of a topological space $X$ is collectionwise normal, then every subset of $X$ is collectionwise normal.

Proof. Let $C$ be a subset of $X$, and let $\left\{H_{\alpha}: \alpha\right.$ in $\left.A\right\}$ be a closed discrete collection in $C$. We want to find a mutually disjoint collection $\left\{V_{\alpha}: \alpha\right.$ in $\left.A\right\}$ of sets open in $C$ such that for all $\alpha$ in $A, H_{\alpha}$ is contained in $V_{\alpha}$. 
For each $\alpha$ in $A$, let

$$
K_{\alpha}=\left(\bigcup_{\beta \neq \alpha} H_{\beta}\right)^{-}
$$

Then for each $\alpha$ in $A, H_{\alpha}$ and $K_{\alpha}$ form a pair of disjoint subsets of $X$. (This assertion follows immediately from the fact that $\left\{H_{\alpha}: \alpha\right.$ in $\left.A\right\}$ is a discrete collection in $C$.) Since $K_{\alpha}$ is a closed set, there is an open set $U_{\alpha}$ such that $H_{\alpha}$ is contained in $U_{\alpha}$, and $U_{\alpha} \cap K_{\alpha}=\varnothing$. Note that for any $\beta \neq \alpha, U_{\alpha} \cap \bar{H}_{\beta}=\varnothing$.

Now let $U$ be the open set $U\left\{U_{\alpha}: \alpha\right.$ in $\left.A\right\}$, and for each $\alpha$ in $A$, let $H_{\alpha}^{\prime}=\bar{H}_{\alpha} \cap U$. Then the collection $\left\{H_{\alpha}^{\prime}: \alpha\right.$ in $\left.A\right\}$ has these properties:

(1) Each $H_{\alpha}^{\prime}$ is closed in $U$.

(2) For each $\alpha$ in $A, H_{\alpha} \subset H_{\alpha}^{\prime} \subset U_{\alpha}$.

(3) For $\alpha \neq \beta, U_{\alpha} \cap H_{\beta}^{\prime}=\varnothing$.

From (1)-(3) it follows that the collection $\left\{H_{\alpha}^{\prime}: \alpha\right.$ in $\left.A\right\}$ is a closed discrete collection in $U$. (Each element of the collection is closed in $U$ by (1); the collection is mutually disjoint since $H_{\alpha}^{\prime} \subset U_{\alpha}$ and $U_{\alpha} \cap H_{\beta}^{\prime}=\varnothing, \beta \neq \alpha$; the collection is locally finite in $U$, since given a point $p$ in $U$, there is an $\alpha$ such that $p$ is in $U_{\alpha}$, and for $\beta \neq \alpha, U_{\alpha} \cap H_{\beta}^{\prime}$ $=\varnothing$.) Since $U$ is collectionwise normal by hypothesis, there is a collection of mutually disjoint open sets $\left\{W_{\alpha}: \alpha\right.$ in $\left.A\right\}$ such that for all $\alpha, H_{\alpha}^{\prime} \subset W_{\alpha}$. Now let $V_{\alpha}=\left(W_{\alpha} \cap C\right)$; then $\left\{V_{\alpha}: \alpha\right.$ in $\left.A\right\}$ is a mutually disjoint collection of sets open in $C$ such that for all $\alpha, H_{\alpha}$ is contained in $V_{\alpha}$.

Lemma 2. Let $X$ be a topological space, and let $\left\{V_{\alpha}: \alpha\right.$ in $\left.A\right\}$ be a locally finite open cover of $X$ such that for all $\alpha$ in $A, \bar{V}_{\alpha}$ is collectionwise normal. Then $X$ is collectionwise normal.

Proof. Let $\left\{H_{\sigma}: \sigma\right.$ in $\left.B\right\}$ be a discrete collection of closed sets in $X$. We want to find a mutually disjoint collection of open sets $\left\{U_{\sigma}: \sigma\right.$ in $\left.B\right\}$ such that for all $\sigma$ in $B, H_{\sigma}$ is contained in $U_{\sigma}$.

For each $\alpha$ in $A$, the collection $\left\{\bar{V}_{\alpha} \cap H_{\sigma}: \sigma\right.$ in $\left.B\right\}$ is a discrete collection of closed sets in $\bar{V}_{\alpha}$. Since $\bar{V}_{\alpha}$ is collectionwise normal by hypothesis, there is a mutually disjoint collection $\left\{G_{\alpha, \sigma}: \sigma\right.$ in $\left.B\right\}$ of sets open in $\bar{V}_{\alpha}$ such that for each $\sigma$ in $B,\left(\bar{V}_{\alpha} \cap H_{\sigma}\right)$ is contained in $G_{\alpha, \sigma}$. We may further assume that the collection $\left\{G_{\alpha, \sigma}: \sigma\right.$ in $\left.B\right\}$ is discrete in $\bar{V}_{\alpha}$ (and hence in $X$ ) by a result due to Nagami [7, p. 84]. Note that for any $\sigma$ in $B, \bar{G}_{\alpha, \sigma}$ is contained in $\bar{V}_{\alpha}$, and that for $\sigma \neq \tau$, $\bar{G}_{\alpha, \sigma} \cap \bar{G}_{\alpha, \tau}=\varnothing$. Put $G=\left\{G_{\alpha, \sigma}: \alpha\right.$ in $A, \sigma$ in $\left.B\right\}$; the collection $G$ is closure preserving by a Lemma due to Michael [6, p. 827]. 
For each $\alpha$ in $A$ and $\sigma$ in $B$, let

$$
H_{\alpha, \sigma}=\bigcup\left\{\bar{G}_{\beta, \tau}: \beta \neq \alpha, \tau \neq \sigma\right\} .
$$

Since $G$ is closure preserving, each $H_{\alpha, \sigma}$ is closed. Now let $U_{\alpha, \sigma}$ $=\left(G_{\alpha, \sigma}-H_{\alpha, \sigma}\right) \cap V_{\alpha}$. The set $U_{\alpha, \sigma}$ is open in $X$. For, $\left(G_{\alpha, \sigma}-H_{\alpha, \sigma}\right)$ is open in $\bar{V}_{\alpha}$, and so $\left(G_{\alpha, \sigma}-H_{\alpha, \sigma}\right) \cap V_{\alpha}$ is open in $V_{\alpha}$, and therefore in $X$; i.e., $U_{\alpha, \sigma}$ is open in $X$.

Finally, for each $\sigma$ in $B$, let

$$
U_{\sigma}=\bigcup_{\alpha \text { in } A} U_{\alpha, \sigma} .
$$

Then the collection $\left\{U_{\sigma}: \sigma\right.$ in $\left.B\right\}$ has these properties and thus is the desired mutually disjoint collection of open sets.

(1) Each $U_{\sigma}$ is an open subset of $X$.

(2) For $\sigma \neq \tau, U_{\sigma} \cap U_{\tau}=\varnothing$.

(3) For all $\sigma$ in $B, H_{\sigma} \subset U_{\sigma}$.

Properties (1) and (2) are clear. We shall prove (3). Let $p$ be a point in $H_{\sigma}$. Since $\left\{V_{\alpha}: \alpha\right.$ in $\left.A\right\}$ covers $X$, there is an $\alpha$ in $A$ such that $p$ is in $V_{\alpha}$. Hence $p$ is in $\left(\bar{V}_{\alpha} \cap H_{\sigma}\right)$, and so $p$ is in $G_{\alpha, \sigma}$. Suppose that for some $\tau \neq \sigma, p$ is in $\bar{G}_{\beta, \tau}$. Then $p$ is in $\bar{V}_{\beta}$, and so $p$ is in $\left(\bar{V}_{\beta} \cap H_{\sigma}\right)$ and therefore is in $G_{\beta, \sigma}$. But $\bar{G}_{\beta, \sigma} \cap \bar{G}_{\beta, \tau}=\varnothing$, a contradiction. Hence $p$ is in $U_{\alpha, \sigma}$ and therefore is in $U_{\sigma}$.

\section{REFERENCES}

1. R. H. Bing, Metrization of topological spaces, Canad. J. Math. 3 (1951), 175-186.

2. J. Dieudonné, Une généralisation des espaces compacts, J. Math. Pures Appl. 23 (1944), 65-76.

3. C. H. Dowker, An embedding theorem for paracompact metric spaces, Duke Math. J. 14 (1947), 639-645.

4. - Inductive dimension of completely normal spaces, Quart. J. Math. 4 (1953), 267-281.

5. E. Michael, $A$ note on paracompact spaces, Proc. Amer. Math. Soc. 4 (1953), 831-838.

6. - Another note on paracompact spaces, Proc. Amer. Math. Soc. 8 (1957), 822-827. $83-88$.

7. K. Nagami, Paracompactness and strong screenability, Nagoya Math. J. 8 (1955),

8. V. Sedivá, On collectionwise normal and hypocompact spaces, Czechoslovak Math. J. 84 (1959), 50-62.

Duke University 